\title{
Uniqueness and Homogeneity of Ordered Relational Structures
}

\section{Citation}

Luce, R. Duncan. 1986. Uniqueness and homogeneity of ordered relational structures. Journal of Mathematical Psychology 30, no. 4: 391-415.

\section{Published Version}

http://dx.doi.org/10.1016/0022-2496(86)90017-9

\section{Permanent link}

http://nrs.harvard.edu/urn-3:HUL.InstRepos:3203652

\section{Terms of Use}

This article was downloaded from Harvard University's DASH repository, and is made available under the terms and conditions applicable to Other Posted Material, as set forth at http:// nrs.harvard.edu/urn-3:HUL.InstRepos:dash.current.terms-of-use\#LAA

\section{Share Your Story}

The Harvard community has made this article openly available.

Please share how this access benefits you. Submit a story.

Accessibility 


\title{
Uniqueness and Homogeneity of Ordered Relational Structures
}

\author{
R. Duncan Luce
}

Harvard University

\begin{abstract}
There are four major results in the paper. (1) In a general ordered relational structure that is order dense, Dedekind complete, and whose dilations (automorphisms with fixed points) are Archimedean, various consequences of finite uniqueness are developed (Theorem 2.6). (2) Replacing the Archimedean assumption by the assumption that there is a homogeneous subgroup of automorphisms that is Archimedean ordered is sufficient to show that the structure can be represented numerically as a generalized unit structure in the sense that the defining real relations satisfy the usual numerical property of homogeneity (Theorem 3.4). The last two results pertain just to idempotent concatenation structures. (3) In a closed, idempotent, solvable, and Dedekind complete concatenation structure, homogeneity is equivalent to the structure satisfying an inductive property analogous to the condition for homogeneity in a positive concatenation structure (Theorem 4.3). Finally, (4) an axiomatization is given for an idempotent structure to be of scale type $(2,2)$, which has previously been shown to be equivalent to a dual bilinear representation. Basically two operations are defined in terms of the given one, and the conditions are that each must be right autodistributive and together they satisfy a generalized bisymmetry property. The paper ends listing several unsolved problems. 1986 Academic Press, Inc.
\end{abstract}

\section{INTRODUCTION}

This paper explores results on uniqueness and homogeneity of real relational structures (Alper, 1984, 1985; Narens, 1981a, 1981b), and it fills in some gaps in the research reported by Luce and Narens (1985) on homogeneous, idempotent, concatenation structures. I shall assume the reader is familiar with these papers except for the unpublished one of Alper (1984). The basic definitions, which may be found in Luce and Narens - in particular, ordered, relational structure, homomorphism,

This work has been supported in part by National Science Foundation Grant IST 83-05819 to Harvard University and by the AT\&T Bell Laboratories. I have benefited from discussions of this work with David H. Krantz and Louis Narens and from comments of Theodore Alper, who pointed out errors in earlier versions. In addition Michael A. Cohen and an anonymous referee made a number of suggestions that are incorporated in this version. I thank each of them. Requests for reprints may be addressed to Department of Psychology, William James Hall, 33 Kirkland Street, Harvard University, Cambridge, MA 02138 . 
and automorphism (Definition 1.1); $M$-point homogeneity, $N$-point uniqueness, and scale type $(M, N)$ (Definition 1.2); and concatenation structure together with various of its properties (Definition 2.1) - will be used here without restatement.

The key result in the sequence of papers by Narens and Alper is this: If an ordered relational structure defined on the real numbers is homogeneous and of finite uniqueness, then it is 2-point unique, the translations (i.e., automorphisms with no fixed point together with the identity) form a group that is of scale type $(1,1)$, and the structure is isomorphic to one for which the automorphisms are a subgroup of the affine group and under that isomorphism the translations map onto the group of all real translations, $x \rightarrow x+s$ where $s \in R e$. The last assertion is sumarized by saying the automorphism group is "conjugate" to a subgroup of the affine group that includes all (real) translations. Part of this development is closely related to Levine (1972), who presented a necessary and sufficient condition for a group of homeomorphisms (strictly increasing transformations from the reals onto the reals) to be transformable into a subset of the affine group, and so to be 2-point unique.

These ideas and results are also closely related to two parts of the mathematical literaturc. One has to do with gencral lincar ordcrings, and some of those references were cited in Luce and Narens (1985). The other, which was pointed out to me by a referee, is work on characterizing subgroups of the general affine group $(x \rightarrow a x+b$, $a \neq 0$ ) which is of concern in geometry. This work focuses on the property of $M$ transitivity, which is like $M$-point homogeneity, but without reference to order. Three relevant papers are Tits (1952(a), 1952(b)) and Burkenhout and Hubaut (1966).

Here I first explore some aspects of the Alper-Narens result from a slightly different perspective with, I believe, some additional insights into the nature of these results. The first major theorem, 2.6, assumes finite uniqueness, order density, and Dedekind completeness, but rather than homogeneity I assume an apparently weaker Archimedean property. Among the things shown is that the commutator subgroup lies within the set of translations and so is 1-point unique.

A sufficient condition for homogeneity is developed (Theorem 3.1) which is applied in Section 4 to idempotent concatenation structures. From Luce and Narens (1985) we know that homogeneous concatenation structures are all closed, and they partition into those that are positivc, negative, and idempotent. If the structure is $N$-point unique for some finite $N$, the first two are of scale type $(1,1)$ and the idempotent ones may be any of the possible types, $(1,1),(1,2)$, or $(2,2)$. (Note that, unlike the results of the preceding sections, these do not depend upon Dedekind completeness.) The positive $(1,1)$ structures are homogeneous PCSs, which are well understood. Narens and Luce (1976, Theorem 2.1) showed that any PCS $\langle X, \gtrsim, 0\rangle$, homogeneous or not, is isomorphic to a numerical PCS and (under a restriction which has since been removed) is 1-point unique. Cohen and Narens (1979) studied the class of homogeneous, Dedekind complete PCSs, showing that each is isomorphic to a unit structure, i.e., a numerical structure $\left\langle\operatorname{Re}^{+}, \geqslant, *, f\right\rangle$, where $*$ is a binary operation on $\operatorname{Re}^{+}$and $f$ is a function from 
$\operatorname{Re}^{+}$such that $f$ is strictly increasing, $f / l$, where $l$ is the identity map, is strictly decreasing, and for all $r, s \in \operatorname{Re}^{+}$,

$$
r * s=s f(r / s) .
$$

In the positive case, $f(1)>1$. Moreover, they showed that in the Dedekind complete case homogeneity is equivalent to the property: each $n$-copy operator is an automorphism, where it will be recalled the $n$-copy operator is defined inductively by $n x=(n+1) x \circ x, 1 x=x$. The key aspect of this condition is that the $n$-copy operator preserves the operation ${ }^{\circ}$. For example, the 2-copy operator condition asserts that for each $x, y \in X$,

$$
(x \circ y) \circ(x \circ y)=(x \circ x) \circ(y \circ y),
$$

which is a universal statement that is a special case of bisymmetry. So the property of homogeneity for PCSs amounts to a countable set of universal statements in the primitives of the system.

Our understanding of the idempotent cases is far less complete. Luce and Narens (1985, Theorem 5.1) showed that a concatenation structure that is closed, idempotent, solvable, and Dedekind complete has a numerical representation and that the structure is 2-point unique. For homogeneous cases, unit representations, now with $f(1)=1$, can again be shown to exist. The three possible scale types were characterized in terms of simple restrictions on the function $f$.

Two results about idempotent structures are missing: first, a criterion for homogeneity in terms of the primitives and, second, an axiomatization for each of the three scale types. Sections 4 and 5 advance our knowledge about the answers to these two questions. Specifically, a criterion is presented for homogeneity (Theorem 4.3) which is similar to, but not quite as satisfactory as, the one for PCSs and, second, an axiomatization is given for the $(2,2)$ case (Theorem 5.1). I do not yet have axiomatizations for the idempotent $(1,1)$ and $(1,2)$ cases.

\section{Uniqueness in Ordered Relational Structures}

\subsection{Uniqueness in General Structures}

Throughout the paper, $\mathscr{X}=\left\langle X, \gtrsim, S_{j}\right\rangle_{j \in J}$ denotes a totally ordered relational structure and $\mathscr{A}$ its group of automorphisms. Any further restrictions will be explicitly noted in the definitions and theorems. We begin with several defined concepts having to do with general relational structures and the subclass of automorphisms that are analogous to real translations.

Definition 2.1. (i) An automorphism $\alpha$ is said to be a dilation at $a$ iff $a \in X$ and $a$ is a fixed point of $\alpha, X$, i.e., $\alpha(a)=a$. The subset of all dilations is denoted by $\mathscr{D}$. The subset of all dilations at $a$, which is easily seen to be a group, is denoted $\mathscr{D}_{a}$. 
(ii) An automorphism is said to be a translation iff either it has no fixed point or it is the identity, $l$; the subset (in general, not a group) of all translations is denoted by $\mathscr{T}$. Note that $\mathscr{D} \cap \mathscr{T}=\{l\}$ and $\mathscr{D} \cup \mathscr{T}=\mathscr{A}$.

(iii) If two relational structures are isomorphic, their automorphism groups are said to be conjugate.

(iv) For $\alpha, \beta \in \mathscr{A}, \alpha \beta \alpha^{-1} \beta^{-1}$ is said to be the commutator of $\alpha$ and $\beta$. The subgroup $\mathscr{C}$ formed by composing finite sequences of commutators is called the commutator (sub)group (Levine, 1972, called it the "derived group").

(iv) The relation $\gtrsim^{\prime}$ is defined on $\mathscr{A}$ as follows: for $\alpha, \beta \in \mathscr{A}, \alpha \succsim^{\prime} \beta$ iff for some nonmaximal $x \in X$ and all $y \succ x, \alpha(y) \succsim \beta(y)$. [It is easy to verify that $\gtrsim^{\prime}$ is transitive since $\succsim$ is, and that $\succsim^{\prime}$ is connected and so a total order if the structure is $N$-point unique (see Corollary 2 of Theorem 2.4).]

(vi) Suppose $\beta \in \mathscr{A}$ and $\beta>^{\prime}$ l. Then $\beta$ is said to be Archimedean in $\mathscr{A}$ iff for each $\alpha \in \mathscr{A}$ there is some integer $n$ such that $\beta^{n} \succ^{\prime} \alpha$.

(vii) $\alpha, \beta \in \mathscr{A}$ are said to be uncrossed iff either $\alpha(x)>\beta(x)$ for all $x \in X$ or $\alpha(x)=\beta(x)$ for all $x \in X$ or $\alpha(x)>B(x)$ for all $x \in X$; otherwise they are said to be crossed. When $\alpha$ and $\imath$ are (un)crossed, $\alpha$ is called (un)crossed. In the uncrossed case, $\alpha \succ^{\prime} \beta$ iff for all $x \in X, \alpha(x)>\beta(x)$. A set of automorphisms is called uncrossed iff each pair of distinct elements from the set is uncrossed. Thus, an uncrossed set of automorphisms is necessarily 1-point unique and so, on that set, $\succsim^{\prime}$ forms a total order.

(viii) A subset $\mathscr{H}$ of $\mathscr{A}$ is said to be convex iff for $\alpha \in \mathscr{H}$ and $\beta \in \mathscr{A}$ if $\alpha \gtrsim^{\prime} \beta$ and $\alpha \succsim^{\prime} \beta^{-1}$, then $\beta \in \mathscr{H}$.

(ix) A subset $\mathscr{H}$ of $\mathscr{A}$ is said to be dense in $\mathscr{X}$ iff for each $x, y \in X$ with $x>y$, there exist $\alpha, \beta \in \mathscr{H}$ such that

$$
x>\alpha(x)>y, \quad x>\beta(y)>y .
$$

TheOREM 2.1. Suppose $\mathscr{X}$ is a totally ordered relational structure. Then

(i) The following are equivalent:

(a) $\mathscr{T}$ is a group under function composition;

(b) $\mathscr{T}$ is 1-point unique;

point.

(c) each dilation, except for the identity, and each translation agree at a

(ii) If $\mathscr{T}$ is nontrivial, then for each $\tau \in \mathscr{T}, \tau \succ^{\prime} l$, the set

$$
\mathscr{G}_{\tau}=\left\{\alpha: \alpha \in \mathscr{A} \text { and for some integer } n, \alpha, \alpha^{-1} \prec^{\prime} \tau^{n}\right\}
$$

is a nontrivial, convex subgroup. 
Proof. (i)(a) implies (b). Suppose for some $x \in X$ and $\alpha, \beta \in \mathscr{T}, \alpha(x)=\beta(x)$. Thus, $x=\alpha^{-1} \beta(x)$ which, since $\alpha^{-1} \beta \in \mathscr{T}$, is possible only if $\alpha^{-1} \beta=l$, proving $\mathscr{T}$ is 1-point unique.

(b) implies (c). Suppose $\alpha$ is a dilation at $x, \alpha \neq l, \tau \in \mathscr{T}$. If they do not intersect, then $\tau^{-1} \alpha \in \mathscr{T}$. However, $\tau^{-1} \alpha(x)=\tau^{-1}(x)$, and so by the 1-point uniqueness of $\mathscr{T}$, $\tau^{-1} \alpha=\tau^{-1}$, whence $\alpha=l$, contrary to choice.

(c) implies (a). Suppose $\mathscr{T}$ is not a group, which is possible under function composition only if it is not closed. Thus, there exists some $\alpha, \beta \in \mathscr{T}$ such that $\alpha \beta \in \mathscr{D}$. By hypothesis (c), there is some $x \in X$ such that $\alpha \beta(x)=\alpha(x)$, whence $\beta(x)=x$, contradicting the assumption that $\beta \in \mathscr{T}$.

(ii) Observe that since $\tau^{-1} \prec^{\prime} \tau \prec^{\prime} \tau^{2}, \tau \in \mathscr{G}_{\tau}$, so $\mathscr{G}_{\tau}$ is nontrivial. It is easy to verify that it is convex. We show it is a group. Since $\imath$ and inverses are in $\mathscr{G}_{\tau}$, it is sufficient to show it is closed. Suppose $\alpha, \beta \in \mathscr{G}$, so for some $m$ and $n, \alpha, \alpha^{-1} \prec^{\prime} \tau^{m}$ and $\beta, \beta^{-1} \prec^{\prime} \tau^{n}$. If $\alpha \prec^{\prime} l$, then $\alpha \beta \prec^{\prime} \imath \beta=\beta \prec^{\prime} \tau^{n}$. If $\beta \prec^{\prime} l$, then $\alpha \beta \prec^{\prime} \alpha l=$ $\alpha \prec^{\prime} \tau^{m}$. If $\alpha, \beta \succsim^{\prime} l$, then for some $a, b \in X$, for $x>a, \alpha(x) \prec \tau^{m}(x)$ and for $x>b$, $\beta(x) \prec \tau^{n}(x)$. Thus, for $x>\max (a, b), \quad \alpha \beta(x) \prec \alpha \tau^{n}(x) \prec \tau^{m} \tau^{n}(x)=\tau^{m+n}(x)$, so $\alpha \beta \prec^{\prime} \tau^{m+n}$. A similar proof holds for $(\alpha \beta)^{-1}=\beta^{-1} \alpha^{-1}$, so $\alpha \beta \in \mathscr{G}_{\tau}$.

COROLLARY. If $\mathscr{X}$ is a totally ordered relational structure and $\mathscr{G} \subseteq \mathscr{T}$ is a group, then $\mathscr{G}$ is 1-point unique.

Proof. Follow that of part (i)(a) implies (b).

From here on, $\mathscr{X}$ will denote a totally ordered relational structure. In each theorem. it is subjected to additional conditions, which are explicitly stated. Whenever $\mathscr{A}$ is assumed to be $N$-point unique, it is implicit that $N$ is finite.

The next two results both show consequences of assuming certain Archimedean properties of the automorphisms. The first involves the assumption of an Archimedean ordered subgroup, and the second, that of each positive dilation being Archimedean.

THEOREM 2.2. Suppose $\mathscr{X}$ is such that $\mathscr{A}$ is $N$-point unique and $\mathscr{G}$ is a subset of $\mathscr{A}$ such that $\left\langle\mathscr{G}, \succsim^{\prime}\right\rangle$ is an Archimedean ordered group.

(i) Then either $\mathscr{G} \subseteq \mathscr{D}$ or $\mathscr{G} \subset \mathscr{T}$.

For $\gamma \in \mathscr{G}$, define

$$
\mathscr{H}=\left\{\alpha: \alpha \in \mathscr{A} \text { and for a positive integer } n, \gamma^{\prime \prime}>^{\prime} \alpha, \alpha^{-1}\right\} .
$$

Then

(ii) $\mathscr{H}$ is independent of the choice of $\gamma$ and it is a convex group.

(iii) $\mathscr{G} \subseteq \mathscr{H}$.

(iv) $\mathscr{G}$ is convex iff $\mathscr{H}=\mathscr{G}$. 
Proof. (i) Suppose otherwise and that $\tau \in \mathscr{G} \cap \mathscr{T}$ and $\alpha \in \mathscr{G} \cap \mathscr{D}, \tau, \alpha \neq i$. Let $a$ denote a fixed point of $\alpha$ and, with no loss of generality, assume $\tau(a) \succ a$. Since $\mathscr{G}$ is Archimedean ordered, $\alpha$ and $\tau$ commute and so $\alpha \tau(a)=\tau \alpha(a)=\tau(a)$. Thus, $\tau(a)$ is another fixed point of $\alpha$. By induction, so are $\tau^{n}(a)$, whence by $N$-point uniqueness, $\tau \equiv l$, contrary to assumption. So one of the two intersections must be $\{l\}$.

(ii) Suppose $\gamma, \eta \in \mathscr{G}, \gamma, \eta \succ^{\prime} l$, and that for some positive integer $n, \gamma^{n} \succ^{\prime} \alpha$, $\alpha^{-1}$. Since $\mathscr{G}$ is Archimedean ordered, for some $m, \eta^{m} \succ^{\prime} \gamma^{n} \succ^{\prime} \alpha, \alpha^{-1}$, and so $\mathscr{H}$ is independent of the choice of $\gamma$. If $\alpha \in \mathscr{H}$ and $\beta \in \mathscr{A}$ and $\alpha>^{\prime} \beta, \beta^{-1}$, then by definition for some $n, \gamma^{n} \succ^{\prime} \alpha \succ^{\prime} \beta, \beta^{-1}$, proving that $\beta \in \mathscr{H}$. Thus, $\mathscr{H}$ is convex. It is easily seen to be a group under function composition since inverses are included by definition and closure is trivial to show.

(iii) Suppose $\alpha \in \mathscr{G}$, then since $\mathscr{G}$ is Archimedean, for some $n, \gamma^{n} \succ^{\prime} \alpha, \alpha^{-1}$, proving $\alpha \in \mathscr{H}$.

(iv) If $\mathscr{G}=\mathscr{H}$, then $\mathscr{G}$ is convex because $\mathscr{H}$ is. Conversely, suppose $\mathscr{G}$ is convex and $\alpha \in \mathscr{H}$. Since $\gamma^{n} \succ^{\prime} \alpha, \alpha^{-1}$ and $\gamma^{n} \in \mathscr{G}$, by the convexity of $\mathscr{G}, \alpha \in \mathscr{G}$. So $\mathscr{H} \subseteq \mathscr{G}$, and by part (iii) we conclude $\mathscr{H}=\mathscr{G}$.

THEOREM 2.3. Suppose $\mathscr{X}$ is such that each positive dilation is Archimedean. Then the following are true:

(i) For each $a \in X,\left\langle\mathscr{D}_{a}, \gtrsim^{\prime}\right\rangle$ is an Archimedean ordered group under function composition.

(ii) If $\mathscr{G}$ is a nontrivial, convex subgroup of $\mathscr{A}$, then either $\mathscr{G}=\mathscr{A}$ or $\mathscr{G} \subseteq \mathscr{T}$.

(iii) If, in addition, $\mathscr{A}$ is $N$-point unique, then the following statements are equivalent:

(a) $\mathscr{A}$ has the property that for each $a, b \in X$ with $a \neq b$ and $\mathscr{D}_{a}$ nontrivial, there is $\beta \in \mathscr{D}_{a}$ such that $\beta(b)>b$,

(b) $\mathscr{A}$ is 2-point unique.

Proof. (i) To show that $\mathscr{D}_{a}$ is a group under function composition, it suffices to note that it is closed: for if $\alpha, \beta \in \mathscr{D}_{a}$, then $\alpha \beta(a)=\alpha(a)=a$, and so $\alpha \beta \in \mathscr{D}_{a}$. It is Archimedean since, by hypothesis, each dilation is Archimedean.

(ii) Suppose $\mathscr{G}-\mathscr{T} \neq \varnothing$, and let $\delta \in \mathscr{G}-\mathscr{T}, \delta \succ^{\prime}$. Since $\delta$ is a dilation, the Archimedean hypothesis implies that, for each $\alpha \in \mathscr{A}$, there exists an integer $n$ such that $\delta^{n} \succ^{\prime} \alpha, \alpha^{-1}$. Since $\mathscr{G}$ is a group and convex, it follows $\alpha \in \mathscr{G}$. So $\mathscr{G}=\mathscr{A}$. Otherwise, $\mathscr{G} \subseteq \mathscr{T}$.

(iii) Suppose $\mathscr{D}_{a}$ is nontrivial. For $a, b \in X$ with $b \succ a$ and $\alpha \in \mathscr{D}_{a}$, suppose $b$ is also a fixed point of $\alpha$. If $\alpha$ is nontrivial, then by hypothesis there exists $\beta \in \mathscr{D}_{a}$ such that $\beta(b)>b$. By part (i) and the fact that an Archimedean ordered group is com- 
mutative, $\alpha \beta(b)=\beta \alpha(b)=\beta(b)$, showing that $\beta(b)(\succ b)$ is also a fixed point of $\alpha$. Since $\beta$ is an automorphism, $\beta^{2}(b) \succ \beta(b)$, and $\alpha \beta^{2}(b)=\beta \alpha \beta(b)=\beta^{2}(b)$, so $\beta^{2}(b)$ also is a fixed point of $\alpha$. By induction, $\alpha$ has $N$ distinct fixed points, and so it is in fact trivial, proving that $\mathscr{X}$ is 2-point unique.

The converse is trivial since if $\alpha \in \mathscr{D}_{a}$ and $\alpha(b)=b, b \neq a$, then by 2-point uniqueness, $\alpha=\imath$.

\subsection{Uniqueness in Order Dense, Dedekind Complete Structures}

THEOREM 2.4. Suppose $\mathscr{X}$ is order dense and Dedekind complete. If $\alpha \in \mathscr{A}$ is crossed, then $\alpha$ is a dilation. In particular, for $x, y \in X$, with $x \prec y$, if either $\alpha(x) \succ x$ and $\alpha(y) \prec y$ or $\alpha(x) \prec x$ and $\alpha(y) \succ y$, then there exists $z \in X, x \prec z \prec y$, such that $\alpha(z)=z$.

Proof. Suppose $\alpha(x) \succ x$ and $\alpha(y) \prec y$. Since $\{z: \alpha(z) \succ z \& z \prec y\}$ is nonempty and is bounded by $y$, the hypothesis that $\mathscr{X}$ is Dedekind complete implies that $u=$ l.u.b. $\{z: \alpha(z)>z \& z<y\}$ exists. Suppose $\alpha(u)>u$, then $u<y$ since $\alpha(y)<y$. By order density, there exists $v$ such that $u \prec v \prec \min [y, \alpha(u)]$. By definition of l.u.b. $\alpha(v) \prec v$. But since $\alpha$ is order preserving, $\alpha(v) \succ \alpha(u)>v$, which is a contradictior. Next suppose $\alpha(u) \prec u$. Since $u$ is a l.u.b. we know there exists $w$ with $\alpha(w) \succ w$ and $\alpha(u) \prec w \prec u$. Thus $\alpha(w) \succ \alpha(u)$ and so $w \succ u$, which is contradiction. So $u$ is a fixed point of $\alpha$. The other case is similar.

Corollary 1. Suppose $\mathscr{X}$ is order dense and Dedekind complete, $\mathscr{G}$ is a subgroup of $\mathscr{A}$, and $\mathscr{G} \subseteq \mathscr{T}$. If $\alpha, \beta \in \mathscr{T}$, then $\alpha$ and $\beta$ are uncrossed and $\mathscr{G}$ is 1-point unique.

Proof. Suppose $\alpha, \beta \in \mathscr{G}$ are such that $\alpha(x)>\beta(x)$ and $\alpha(y)\langle\beta(y)$. So $x>$ $\alpha^{-1} \beta(x)$ and $y \prec \alpha^{-1} \beta(y)$. By the Theorem $\alpha^{-1} \beta$ has a fixed point which, since $\alpha^{-1} \beta$ is not the identity, is imposible because $\mathscr{G} \subseteq \mathscr{T}$. Thus, $\alpha$ and $\beta$ are uncrossed. By the Corollary to Theorem $2.1, \mathscr{G}$ is 1 -point unique.

Corollary 2. Suppose $\mathscr{X}$ is order dense, Dedekind complete, and N-point unique. If $\alpha \in \mathscr{D}$, then for some $a \in X, \alpha \in \mathscr{D}_{a}$, and either $\alpha(x)>x$ for all $x>a$ or $\alpha(x) \prec x$ for all $x>a$.

Proof. Since $\mathscr{X}$ is $N$-point unique, any $\alpha \neq \imath$ has at most $N-1$ fixed points, so there is a largest, $a$, in which case $\alpha \in \mathscr{D}_{a}$. Suppose, for some $x, y>a$ we have $\alpha(x)>x$ and $\alpha(y)<y$. By the proof of the theorem there is a fixed point between $x$ and $y$, which contradicts that $a$ is the largest fixed point.

Theorem 2.5. Suppose $\mathscr{X}$ is order dense, Dedekind complete, and $N$-point unique. Then each dilation is Archimedean in $\mathscr{A}$ iff for each $\alpha \in \mathscr{A}, \alpha \succ^{\prime}$, and $\beta \in \mathscr{D}, \beta \succ^{\prime}{ }^{\prime}$, the set of fixed points of $\left\{\beta^{n} \alpha^{-1}: n\right.$ an integer $\}$ is bounded from above.

Proof. Suppose $\beta \in \mathscr{D}, \beta \succ^{\prime}$ l, is Archimedean. So for $\alpha \in \mathscr{A}$, there is some integer $n$ such that $\beta^{n} \succ^{\prime} \alpha$, i.e., $\beta^{n} \alpha^{-1} \succ^{\prime} \imath$. Since $\mathscr{X}$ is $N$-point unique, $\beta^{n} \alpha^{-1}$ has 
either no fixed point or a maximal one. Let $a$ denote the maximum of the fixed points of $\beta$ and $\beta^{n} \alpha^{-1}$, then for $x>a, \beta^{n} \alpha^{-1}(x) \succ x$. Thus, for any $m>n$,

$$
\beta^{m} \alpha^{-1}(x)=\beta^{m-n} \beta^{n} \alpha^{-1}(x) \succ \beta^{m-n}(x) \succ x,
$$

and so all have their maximum fixed point $\lesssim a$. Since there are only $n-1$ other $\beta^{i} \alpha^{-1}, i=1, \ldots, n-1$, each having a maximal fixed point or none, the set of all maximal fixed points is bounded from above.

Conversely, suppose the set of fixed points is bounded from above and that $\beta$ is not Archimedean. This means that for some $\alpha \in \mathscr{A}, \alpha \gtrsim^{\prime} \beta^{n}$ holds for all $n$, so $i \gtrsim^{\prime} \beta^{n} \alpha^{-1}$. Select $b$ to be upper bound on the fixed points of $\left\{\beta^{n} \alpha^{-1}\right\}$. Thus, for all $n$ and all $y>b, \beta^{n} \alpha^{-1}(y) \lesssim y$, and so for all $x>a=\alpha^{-1}(b), \beta^{n}(x) \lesssim \alpha(x)$, i.e., $\alpha(x)$ bounds $\left\{\beta^{n}(x): n\right.$ an integer $\}$. By Dedekind completeness, for each $x>a$ there is a l.u.b $u(x)$. Since $\beta \succ^{\prime} u, \beta u(x) \succsim u(x)$ for all $x>a$. Suppose $\beta u(x) \succ u(x)$, then for each $n$ we have $\beta u(x)>u(x) \succsim \beta^{n}(x)$, and so taking $\beta^{-1}, u(x)>\beta^{-1} u(x) \succsim$ $\beta^{n-1}(x)$, showing that $\beta^{-1} u(x)$ is a smaller bound than $u(x)$, contrary to choice. Thus, $u(x)$ is a fixed point of $\beta$, and so by $N$-point uniqueness $\beta=l$, contrary to choice.

Part (ii) of the following result is due to Alper (1984); otherwise it appears to be new although closely related to the previous work.

THEOREM 2.6. Suppose $\mathscr{X}$ is order dense and Dedekind complete structure, $\mathscr{A}$ is $N$-point unique, and each dilation is Archimedean. Then the following are true:

(i) Either $\left\langle\mathscr{A}, \succsim^{\prime}\right\rangle$ is an Archimedean ordered group under function composition or there exists a unique, nontrivial, convex group $\mathscr{G}$ such that $\mathscr{G} \subseteq \mathscr{T}$ and $\left\langle\mathscr{G}, \gtrsim^{\prime}\right\rangle$ is Archimedean ordered.

(ii) If the group $\mathscr{G}$ of part (i) exists, then the commutator group $\mathscr{C} \subseteq \mathscr{G}$.

(iii) If $\mathscr{A}$ is 2-point unique and $\mathscr{T}$ is a group, then $\mathscr{T}$ is convex.

Proof. (i) Suppose $\mathscr{G}$ and $\mathscr{H}$ are nontrivial, convex, proper subgroups of $\mathscr{A}$ with $\mathscr{G} \neq \mathscr{H}$, then with no loss of generality there exists $\alpha \in \mathscr{H}-\mathscr{G}$ with $\alpha \succ^{\prime} \imath$. Since $\mathscr{G}$ is nontrivial, select $\beta \in \mathscr{G}$ with $\beta \succ^{\prime}$ l. Observe that for every integer $n$, $\alpha \succ^{\prime} \beta^{n}$ since otherwise

$$
\beta^{n} \succ^{\prime} \alpha \succ^{\prime} \iota \succ^{\prime} \alpha^{-1},
$$

and by the convexity of $\mathscr{G}, \alpha \in \mathscr{G}$ contrary to choice. By Theorem 2.3 (ii), $\mathscr{H} \subseteq \mathscr{G}$, and so by Corollary 1 to Theorem 2.4, $\alpha$ and $\beta^{n}$ are uncrossed. Thus, for each $x \in X$, $\alpha(x)>\beta^{n}(x)$. So $u(x)=$ l.u.b. $\left\{\beta^{n}(x): n\right.$ an integer $\}$ exists. Suppose $\beta u(x) \succ u(x)$, then since $u(x) \succsim \beta^{n}(x)$ we see by taking $\beta^{-1}$ that $u(x) \succ \beta^{-1} u(x) \succsim \beta^{n-1}(x)$. Thus, $\beta^{-1} u$ is a smaller bound than $u$, contrary to choice. So $u(x)$ is a fixed point of $\beta$, which by $N$-point uniqueness means $\beta=\imath$, contrary to choice. So $\mathscr{H} \subseteq \mathscr{G}$. Similarly, $\mathscr{G} \subseteq \mathscr{H}$, whence $\mathscr{G}=\mathscr{H}$.

If $\mathscr{A}=\mathscr{D}$, then by hypothesis it is Archimedean ordered. So, suppose $\mathscr{T}$ is nontrivial and $\tau \in \mathscr{T}, \tau \succ^{\prime}$ l. Let $\mathscr{G}_{\tau}$ be the group defined in Theorem 2.1. By 
Theorems 2.1(ii) and 2.3(ii), $\mathscr{G}_{\tau}$ is nontrivial and convex and either $\mathscr{G}_{\tau}=\mathscr{A}$ or $\mathscr{G}_{\tau} \subseteq \mathscr{T}$. Suppose, first, that for all $\tau \in \mathscr{T}, \tau \succ^{\prime} \imath, \mathscr{G}_{\tau}=\mathscr{A}$. Then by the definition of these groups and by the hypothesis that dilations are Archimedean, $\left\langle\mathscr{A}, \succsim^{\prime}\right\rangle$ is Archimedean ordered. So assume at least one, call it $\mathscr{G}$, is a subgroup of $\mathscr{T}$. It is, as we have shown, unique. Using the same l.u.b. argument as above, we see $\left\langle\mathscr{G}, \succsim^{\prime}\right\rangle$ is Archimedean ordered.

(ii) Suppose $\alpha, \beta \in \mathscr{A}$. If $\mathscr{G}=\mathscr{A}$, the result is trivial. Suppose otherwise, then by what we have shown in part (i), we know $\mathscr{G}$ is convex and by its uniqueness, just shown, no other convex group lies properly between $\mathscr{G}$ and $\mathscr{A}$. Thus, according to Fuchs $(1966$, p. 50) $\mathscr{A} / \mathscr{G}=\{\alpha \mathscr{G}: \alpha \in \mathscr{A}\}$ with the group operation defined by $(\alpha \mathscr{G})(\beta \mathscr{G})=\alpha \beta \mathscr{G}$ is isomorphic to a subgroup of the additive real numbers. Thus it is commutative and so $\alpha \beta \mathscr{G}=\beta \alpha \mathscr{G}$. Therefore,

$$
\begin{aligned}
\mathscr{G} & =(\beta \alpha)^{-1}(\beta \alpha) \mathscr{G} \\
& =(\beta \alpha)^{-1}(\beta \alpha \mathscr{G}) \\
& =(\beta \alpha)^{-1}(\alpha \beta \mathscr{G}) \\
& =\left(\alpha^{-1} \beta^{-1} \alpha \beta\right) \mathscr{G} .
\end{aligned}
$$

and so $\alpha^{-1} \beta^{-1} \alpha \beta \in \mathscr{G}$, whence $\mathscr{C} \subseteq \mathscr{G}$.

(iii) Assume $\tau \in \mathscr{T}, \tau \succ^{\prime} \imath, \mathscr{T}$ is a group, and $\alpha \in \mathscr{D}, \alpha \succ^{\prime}$. We show that $\alpha \succ^{\prime} \tau$, which proves $\mathscr{T}$ is convex. Suppose, on the contrary, $\tau \gtrsim^{\prime} \alpha$. By Theorem 2.1(i), $\alpha$ and $\tau$ agree at some point $a$ and, by 2-point uniqueness, only at $a$. Thus, from Theorem 2.4 and the definition of $\succsim^{\prime}$, we see that for $x>a, \alpha(x) \prec \tau(x)$, and for $x \prec a, \alpha(x) \succ \tau(x) \succ x$, for otherwise there would be a fixed point different from $a$. Since $\alpha$ and $\tau^{-1}$ intersect at some point $b$ and $\tau^{-1}(x) \prec x$ for all $x$, it follows that $b \succ a$. Thus by Theorem 2.4, for some $c, a \prec c \prec b, \alpha(c)=c$. Since $\alpha \succ^{\prime} l$, for some $d>b$ and all $x>d, \alpha(x)>x$. Thus, by Theorem 2.4, there is some $e$ with $b<e<d$ such that $\alpha(e)=e$. By 2-point uniqueness, $\alpha=1$, contrary to assumption. So $\mathscr{T}$ is convex.

Theorem 2.6 is useful only to the extent that one can understand structurally when the dilations are all Archimedean. As we have seen in Theorem 2.5, this is equivalent to an upper bound on the fixed points of $\left\{\beta^{n} \alpha^{-1}\right\}$, but $I$ have been unable to find structural conditions that insure this property. Once that is understood, it will become reasonably clear when finite uniqueness really means $N \leqslant 2$. As Alper (1984) has shown, a sufficient condition is homogeneity. I do not know of a weaker condition, but almost certainly some exist.

In the language introduced by Narens $(1981 \mathrm{~b})$, the facts that $\mathscr{G} \subseteq \mathscr{T}$ and $\mathscr{G}$ is convex (part (i)) mean that the elements of $\mathscr{G}$ are infinitesimal relative to each dilation, i.e., if $\alpha \in \mathscr{D}, \alpha \succ^{\prime} l$, and $\tau \in \mathscr{G}, \tau \succ^{\prime} l$, then for each integer $n, \alpha \succ^{\prime} \tau^{n}$. For, if not, then $\alpha \in \mathscr{G}$ and so is a translation, contrary to choice. 


\section{Homogeneity in Order Dense, Dedekind Complete Structures}

\subsection{Homogeneity in General Structures}

The next rcsult describes a sufficient condition for homogeneity in order dense, Dedekind complete structures. We will use this result later in studying idempotent, concatenation structures.

THEOREM 3.1. Suppose $\mathscr{X}$ is order dense and Dedekind complete, and suppose $\mathscr{G}$ is a subgroup of $\mathscr{A}$ for which $\mathscr{G} \subseteq \mathscr{T}$. If $\left\langle\mathscr{G}, \succsim^{\prime}\right\rangle$ is Dedekind complete and $\mathscr{G}$ is dense in $\mathscr{X}$, then $\mathscr{X}$ is homogeneous.

Proof. Since by Corollary 1 to Theorem $2.4, \mathscr{G}$ is uncrossed, $Z^{\prime}$ is a total order on $\mathscr{G}$. Suppose $x, y \in X$. Define

$$
\begin{aligned}
\mathscr{B} & =\{\tau: \tau \in \mathscr{G} \text { and } \tau(x) \succsim y\} \\
\mathscr{B}^{\prime} & =\{\tau: \tau \in \mathscr{G} \text { and } \tau(x) \prec y\} .
\end{aligned}
$$

If $x \prec y$, then by the density of $\mathscr{G}$ in $\mathscr{X}$, there exists $\tau^{\prime} \in \mathscr{G}$ such that $x \prec \tau^{\prime}(x) \prec y$. So $\imath \prec^{\prime} \tau^{\prime}$. For some integer $n, y \prec \tau^{\prime n}(x)$, for otherwise by Dedekind completeness $\tau^{\prime}$ has a fixed point, contrary to the assumption that $\mathscr{G} \subseteq \mathscr{T}$. So $\mathscr{B}$ and $\mathscr{B}{ }^{\prime}$ are both nonempty. The argument is similar for $x\rangle y$. Since $\left\langle\mathscr{G}, \succsim^{\prime}\right\rangle$ is Dedekind complete, there is a cut element $\delta$. Suppose $\delta(x) \succ y$, then by the density of $\mathscr{G}$ in $\mathscr{A}$, there is $\alpha \in \mathscr{G}$ such that $\delta(x) \succ \alpha[\delta(x)] \succ y$. Since $\succsim^{\prime}$ is a total order, $\delta \succ^{\prime} \alpha \delta$, which together with $\alpha \delta \in \mathscr{G}$ contradicts the choice of $\delta$ as the cut element. A similar argument shows $\delta(x) \prec y$ is impossible. So $\delta(x)=y$, proving that $X$ is homogeneous.

\subsection{Homogeneity in $N$-point Unique Structures}

We now turn to results that depend upon $N$-point uniqueness as well as homogeneity.

Theorem 3.2. Suppose $\mathscr{X}$ is such that $\mathscr{A}$ is $N$-point unique and $\mathscr{G}$ is an Archimedean ordered subgroup of automorphisms that is homogeneous. Then,

(i) $\mathscr{G} \subseteq \mathscr{T}$ and so $\mathscr{G}$ is 1-point unique.

(ii) With $\mathscr{H}$ defined as in Theorem 2.2, the following are equivalent:

(a) $\mathscr{H}=\mathscr{G}$,

(b) $\mathscr{H} \subseteq \mathscr{T}$,

(c) $\mathscr{H}$ is 1-point unique.

Proof. (i) By Theorem 2.2(i) we know that either $\mathscr{G} \subseteq \mathscr{D}$ or $\mathscr{G} \subseteq \mathscr{T}$. Suppose the former, and let $a$ be a fixed point of some $\alpha \in \mathscr{G}$. Consider any $\beta \in \mathscr{G}$ and suppose $b$ 
is one of its fixed points. By the homogeneity of $\mathscr{G}$, there exists $\gamma \in \mathscr{G}$ such that $\gamma(a)=b$. Since $\mathscr{G}$ is commutative,

$$
\gamma \beta(a)=\beta \gamma(a)=\beta(b)=b=\gamma(a),
$$

so by applying $\gamma^{-1}$ we see $a$ is a fixed point of $\beta$, which means that $\mathscr{G}$ cannot be homogeneous, contrary to assumption. So $\mathscr{G} \subseteq \mathscr{T}$. By the Corollary to Theorem 2.1, $\mathscr{G}$ is 1 -point unique.

(ii)(a) implies (b) by part (i).

(ii)(b) implies (c) by the corollary to Theorem 2.1 .

(ii)(c) implies (a). Suppose $\alpha \in \mathscr{H}$ and $x \in X$. By the homogeneity of $\mathscr{G}$, there is $\beta \in \mathscr{G}$ such that $\beta(x)=\alpha(x)$. Since by Theorem 2.2(iii), $\mathscr{G} \subseteq \mathscr{H}$, and $\mathscr{H}$ is 1 -point unique, we can conclude $\alpha \equiv \beta$, and so $\alpha \in \mathscr{G}$. So $\mathscr{H}=\mathscr{G}$.

For the following result, recall $\mathscr{D}$ is the set of dilations and $\mathscr{C}$ is the commutator group.

THEOREM 3.3. Suppose $\mathscr{X}$ is order dense and Dedekind complete and $\mathscr{A}$ is homogeneous and $N$-point unique. If $\mathscr{D}$ is nontrivial, then

(i) $\mathscr{D}$ is homogeneous, and

(ii) $\mathscr{C}$ is homogeneous and noncyclic.

Proof. (i) Let $x, y \in X$. The proof is given for $x>y$; a similar one follows for $x<y$. First, we show that there is a dilation $\alpha$ at $x$ such that for all $u>x, \alpha(u) \neq u$. Let $\delta$ be a nontrivial dilation with a maximal fixed point at, say, $z$. Such exists because, by hypothesis, there is a nontrivial dilation, and if it had no maximal fixed point, then by $N$-point uniqueness it would in fact be the identity. By homogeneity, there is $\beta \in \mathscr{A}$ such that $\beta(z)=x$. Let $\alpha=\beta \delta \beta^{-1}$. Then

$$
\alpha(x)=\beta \delta \beta^{-1}(x)=\beta \delta(z)=\beta(z)=x,
$$

and for $u \succ x, \alpha(u) \neq u$ since, otherwise, $\delta \beta^{-1}(u)=\beta^{-1}(u)$ and $\beta^{-1}(u) \succ \beta^{-1}(x)=z$, which violates the choice of $z$ as the maximal fixed point of $\delta$.

Next, for any $w>x$, we show there is a dilation $\beta$ at $x$ with $\beta(y)>w$. We know there exists a dilation $\alpha$ at $x$ such that for all $u \succ x, \alpha(u) \neq u$. By Theorem 2.4 and using either $\alpha$ or $\alpha^{-1}$, there is no loss of generality in assuming $\alpha(u) \succ u$. So we know, $u<\alpha(u)<\alpha^{2}(u)<\cdots$. If this sequence were bounded, then by the fact the structure is Dedekind complete, there will be a fixed point of $\alpha$ greater than $x$, contrary to choice. Thus, for some $n, \beta=\alpha^{n}$ has the asserted property.

Now we show there is $\alpha \in \mathscr{D}$ such that $\alpha(x)=y$. By 1 -point homogeneity, there exists $\beta \in \mathscr{A}$ such that $\beta(x)=y$. If $\beta$ is a dilation, we are done. Otherwise, $\beta \in \mathscr{T}$, and since $y \succ x$, it follows from Theorem 2.4 that $\beta(u) \succ u$ for all $u$. Select $z>\beta(y)$ and let $\gamma$ be a dilation at $x$ with $\gamma(y)>z$. So $\gamma^{-1}(z)<y$. Consider $\alpha=\beta \gamma^{-1}$. Observe that $\alpha(x)=\beta \gamma^{-1}(x)=\beta(x)=y$. To show that $\alpha$ is a dilation, observe that 
$\alpha(z)=\beta \gamma^{-1}(z) \prec \beta(y) \prec z$ and $\alpha(x)=y \succ x$, so by Theorem 2.4, it must be a dilation.

(ii) By part (i), since $\mathscr{D}$ is nontrivial, it is homogeneous. So for $x, y \in X$, there exists $\beta \in \mathscr{D}$ such that $\beta(x)=y$. Let $z$ be a fixed point of $\beta$, then there exists $\alpha \in \mathscr{A}$ such that $\alpha(y)=z$. Thus

$$
\alpha^{-1} \beta^{-1} \alpha \beta(x)=\alpha^{-1} \beta^{-1} \alpha(y)=\alpha^{-1} \beta^{-1}(z)=\alpha^{-1}(z)=y,
$$

which establishes that $\mathscr{C}$ is homogeneous.

If $\mathscr{C}$ were cyclic, then each of its elements would be of the form $\tau^{n}$ for some fixed $\tau \in \mathscr{C}$. But since an order dense, Dedekind complete structure has uncountably many elements and $\left\{\tau^{n}(x)\right\}$ is countable, $\mathscr{C}$ cannot be homogeneous, contrary to what has just be shown. So $\mathscr{C}$ is not cyclic.

Corollary. Suppose $\mathscr{X}$ is order dense and Dedekind complete, $\mathscr{A}$ is homogeneous and $N$-point unique, and $\mathscr{D}$ is nontrivial and each of its members is Archimedean. Then $\mathscr{C}$ is the unique, proper, convex, Archimedean ordered subgroup of $\mathscr{A}$.

Proof. By Theorem 2.6(i) and (ii) and Theorem 3.2 there exists a unique, proper, convex, Archimedean ordered subgroup $\mathscr{G}$ such that $\mathscr{C} \subseteq \mathscr{G} \subseteq \mathscr{T}$. Suppose $\gamma \in \mathscr{G}-\mathscr{C}$ and $x \in X$. By the present theorem, $\mathscr{C}$ is homogeneous, so there exists $\tau \in \mathscr{C}$ such that $\tau(x)=\gamma(x)$. By Corollary 1 to Theorem 2.4, $\tau \equiv \gamma$, contradicting the choice of $\gamma$. So $\mathscr{C}=\mathscr{G}$.

\subsection{Generalized Unit Structures}

The following theorem, which was suggested to me by Louis Narens, generalizes the result that homogeneous positive concatenation structures have representations as unit structures (Cohen \& Narens, 1979, Theorem 3.3). A unit structure, it will be recalled, is simply a positive concatenation structure defined on $\mathrm{Re}^{+}$for which the operation $\circ$ is homogeneous in the usual sense of functions, i.e., for all $r, s, t \in \operatorname{Re}^{+}$, $r s \circ r t=r(s \circ t)$. We may generalize this concept as follows: Let $R \subseteq \operatorname{Re}^{+}$be closed under multiplication. A relation $R_{j}$ of order $n$ on $R$ is said to be homogeneous iff for every $r_{i}, s \in R, i=1, \ldots, n$,

$$
\left(r_{1}, \ldots, r_{n}\right) \in R_{j} \quad \text { iff } \quad\left(s r_{1}, \ldots, s r_{n}\right) \in R_{j} .
$$

THeOREM 3.4. Suppose $\mathscr{X}$ is such that $\mathscr{A}$ includes a homogeneous subgroup of translations that is Archimedean ordered under $\gtrsim^{\prime}$. Then there exists a homogeneous relational structure in $\mathrm{Re}^{+}, \mathscr{R}$, such that $\mathscr{X}$ is isomorphic to $\mathscr{R}$.

Proof. Let $\mathscr{G}^{\prime}=\left\langle\mathscr{G}, \succsim^{\prime}, *\right\rangle$, where $*$ denotes function composition, be the Archimedean ordered, homogeneous subgroup of $\mathscr{T}$. We first imbed $\mathscr{X}$ isomorphically in $\left\langle\mathscr{G}, \succsim^{\prime}\right\rangle$. Let $n(j)=\operatorname{order}\left(S_{j}\right)$. For $\alpha_{i} \in \mathscr{G}, i=1, \ldots, n(j)$, and for a fixed $x \in X$, define $S_{j}^{\prime}$ on $\mathscr{G}$ by

$$
\left(\alpha_{1}, \ldots, \alpha_{n(j)}\right) \in S_{j}^{\prime}, \quad \text { iff } \quad\left(\alpha_{1}(x), \ldots, \alpha_{n(j)}(x)\right) \in S_{j} .
$$


Note that the definition of $S_{j}^{\prime}$ is independent of the choice of $x$. For suppose we had chosen $y \in X$, then by the fact that $\mathscr{G}$ is homogeneous we know there exists $\beta \in \mathscr{G}$ such that $y=\beta(x)$. Using this, the fact that $\beta$ is an automorphism and so is invariant under the defining relations $S_{j}$, and the fact that by Hölder's theorem elements of $\mathscr{G}$ commute we have

$$
\begin{array}{lll}
\left(\alpha_{1}(x), \ldots, \alpha_{n(j)}(x)\right) \in S_{j} \quad \text { iff } & \left(\beta \alpha_{1}(x), \ldots, \beta \alpha_{n(j)}(x)\right) \in S_{j} \\
& \text { iff } \quad\left(\alpha_{1} \beta(x), \ldots, \alpha_{n(j)} \beta(x)\right) \in S_{j} \\
& \text { iff } \quad\left(\alpha_{1}(y), \ldots, \alpha_{n(j)}(y)\right) \in S_{j} .
\end{array}
$$

For fixed $x$, define the function $F$ from $\mathscr{G}$ into $X$ by: for each $\alpha \in \mathscr{G}$,

$$
F(\alpha)=\alpha(x) .
$$

It is onto $X$ because $\mathscr{G}$ is homogeneous, and it is $1: 1$ because $\mathscr{G}$ is 1 -point unique. We show that the elements of $\mathscr{G}$ are uncrossed. For suppose otherwise and there exist $\gamma \in \mathscr{G}$ and $x, y \in X$ such that $\gamma(x) \succ x$ and $\gamma(y)<y$. By the homogeneity of $\mathscr{G}$, there exists $\sigma \in \mathscr{G}$ such that $\sigma(x)=y$. Since $\mathscr{G}$ is Archimedean ordered, its elements commute and so,

$$
\sigma \gamma(x)>\sigma(x)=y>\gamma(y)=\gamma \sigma(x)=\sigma \gamma(x),
$$

which is impossible. Thus,

$$
\alpha \succsim^{\prime} \beta \quad \text { iff } \quad \alpha(x) \succsim \beta(x) \quad \text { iff } \quad F(\alpha) \succsim F(\beta) .
$$

Finally,

$$
\begin{array}{ll}
\left(\alpha_{1}, \ldots, \alpha_{n(j)}\right) \in S_{j}^{\prime} \quad \text { iff } \quad\left(\alpha_{1}(x), \ldots, \alpha_{n(j)}(x)\right) \in S_{j} \\
\text { iff } \quad\left(F\left(\alpha_{1}\right), \ldots, F\left(\alpha_{n(j)}\right)\right) \in S_{j} .
\end{array}
$$

Thus, $F$ is the isomorphism asserted.

Let $\varphi$ denote the isomorphism between $\mathscr{G}^{\prime}$ and $\langle R, \geqslant, \cdot\rangle, R \subseteq \operatorname{Re}^{+}$(Hölder's theorem). Define the relation $R_{j}$ of order $n(j)$ on $R$ by: for $r_{i} \in R, i=1, \ldots, n(j)$,

$$
\left(r_{1}, \ldots, r_{n(,)}\right) \in R_{j} \quad \text { iff } \quad\left(\varphi^{-1}\left(r_{1}\right), \ldots, \varphi^{-1}\left(r_{n(,)}\right)\right) \in S_{j}^{\prime} .
$$

It is easy to verify that $\left\langle\mathscr{G}, \succsim^{\prime}, S_{j}^{\prime}\right\rangle_{j \in J}$ and $\left\langle R, \geqslant, R_{j}\right\rangle_{j \in J}$ are isomorphic. We show that $R_{j}$ is homogeneous by using the fact that $\varphi$ maps function composition onto multiplication and the fact that automorphisms are invariant under $S_{j}^{\prime}$,

$$
\begin{aligned}
\left(r_{1}, \ldots, r_{n(j)}\right) \in R_{j} \quad \text { iff } & \left(\varphi^{-1}\left(r_{1}\right), \ldots, \varphi^{-1}\left(r_{n(j)}\right)\right) \in S_{j}^{\prime} \\
\text { iff } & \left(\varphi^{-1}(s) * \varphi^{-1}\left(r_{1}\right), \ldots, \varphi^{-1}(s) * \varphi^{-1}\left(r_{n(j)}\right)\right) \in S_{j}, \\
\text { iff } & \left(\varphi^{-1}\left(s r_{1}\right), \ldots, \varphi^{-1}\left(s r_{n(j)}\right)\right) \in S_{j}^{\prime} \\
\text { iff } & \left(s r_{1}, \ldots, s r_{n(j)}\right) \in R_{j} .
\end{aligned}
$$




\section{Homogeneity of Closed, Idempotent, Solvable, Dedekind Complete Concatenation Structures}

\subsection{Preliminary Results}

DEFINITION 4.1. Suppose $\mathscr{X}$ is a concatenation structure. The operation is said to be lower (upper) semicontinuous iff for each $x, y, z \in X$ for which $x \circ y$ is defined and $z \prec x \circ y(z \succ x \circ y)$ there exists $x^{\prime}, y^{\prime} \in X$ such that $x^{\prime} \prec x, y^{\prime} \prec y$, and $z \prec x^{\prime} \circ y^{\prime}$ $\left(x^{\prime} \succ x, y^{\prime} \succ y, z \succ x^{\prime} \circ y^{\prime}\right)$.

The first result, which was pointed out to me by Michael A. Cohen, establishes a useful sufficient condition for semicontinuity.

LEMMA 4.1. Suppose $\mathscr{X}$ is a concatenation structure that is closed, solvable, order dense, and Dedekind complete. Then upper and lower semicontinuity hold.

Proof. As the two halves are similar, we show only lower semicontinuity. Suppose $z \prec x \circ y$. By order density, there exists $u$ such that $z \prec u \prec x \circ y$. By order density and Dedekind completeness, we can find an increasing sequence $\left\{x_{i}\right\}$ such that for every $i, x_{i} \prec x$ and l.u.b. $\left\{x_{i}\right\}=x$. For each $i$, let $y_{i}$ solve $u=x_{i} \circ y_{i}$ and let $y^{*}$ solve $u=x \circ y^{*}$. Since $u \prec x \circ y, y^{*}<y$. Observe that $\left\{y_{i}\right\}$ is necessarily a decreasing sequence with g.l.b $y^{*}$. So, for sufficiently large $i, y^{*}<y_{i}<y$. And so $x_{i} \circ y_{i}$ fulfills the condition since by construction and choice, $x_{i} \prec x, y_{i} \prec y$, and $z \prec u=x_{i} \circ y_{i} \prec x \circ y$.

THEOREM 4.1. Suppose $\mathscr{X}$ is a concatenation structure that is closed, idempotent, solvable, and Dedekind complete, and $\mathscr{G}$ is a maximal group included in $\mathscr{T}$. Then $\left\langle\mathscr{G}, \succsim^{\prime}\right\rangle$ is Dedekind complete.

Proof. Let $\Gamma$ be a bounded subset of $\mathscr{G}$. Since by Corollary 1 to Theorem 2.4 , $\succsim^{\prime}$ is a total ordering, the bound of $\Gamma$ yields a bound of $\{a(x): \alpha \in \Gamma\}$ for each $x \in X$. So, $\mu(x)=$ l.u.b. $\{\alpha(x) \alpha \in \Gamma\}$ is defined. We show that $\mu$ is in $\mathscr{G}$.

(1) $\mu(x \circ y)=\mu(x) \circ \mu(y)$. Suppose not. If $\mu(x \circ y) \succ \mu(x) \circ \mu(y)$, then since $\mu$ is a l.u.b., there exists $\alpha \in \Gamma$ such that

$$
\mu(x \circ y) \succsim \alpha(x \circ y) \succ \mu(x) \circ \mu(y) \succsim \alpha(x) \circ \alpha(y)=\alpha(x \circ y),
$$

which is impossible. Suppose the other inequality. By the fact that idempotence implies order density, Lemma 4.1 shows that lower semicontinuity holds. So there exist $x^{\prime}, y^{\prime} \in A$ such that $x^{\prime}<\mu(x), y^{\prime}<\mu(y)$, and $\mu(x \circ y)<x^{\prime} \circ y^{\prime}$. Thus, there exist $\alpha, \beta \in \Gamma$ with $x^{\prime}\left\langle\alpha(x) \lesssim \alpha(x) \lesssim \mu(x)\right.$ and $y^{\prime} \prec \beta(y) \lesssim \mu(y)$. But by Corollary 1 to Theorem 2.4, $\mathscr{G}$ is ordered, so select the larger of $\alpha$ and $\beta$, say $\alpha$, then we have

$$
\mu(x) \circ \mu(y) \succsim \alpha(x) \circ \alpha(y)=\alpha(x \circ y) \succ x^{\prime} \circ y^{\prime} \succ \mu(x \circ y),
$$

which is impossible since $\mu(x \circ y) \succsim \alpha(x \circ y)$. 
(2) $\mu$ is order preserving. Suppose, on the contrary $x>y$ and $\mu(x)=\mu(y)$. By solvability, there exists a nontrivial standard difference sequence $\left\{z_{i}\right\}$ such that $z_{i} \circ y=z_{i+1} \circ x$. By part 1 and monotonicity, $\mu\left(z_{i}\right)=\mu\left(z_{i+1}\right)$. Let $u$ denote this common value. By the choice of $\Gamma$ there exists some $\tau \in \mathscr{G}$ such that $\tau \gtrsim^{\prime} \alpha$ for all $\alpha \in \Gamma$, i.e, $\tau(x) \succsim \alpha(x)$ is true independent of $x$. Thus, $\tau\left(z_{i}\right) \succsim \mu\left(z_{i}\right)=u$. But since $X$ is Archimedean (Luce \& Narens, 1985, Theorem 2.1) and $\tau$ is an automorphism, it follows that $\tau(x) \succsim u$ for all $x \in X$. Because $\mathscr{X}$ is solvable, there is no minimal element, which means $\tau$ is not onto, contradicting the assumption it is in $\mathscr{G}$. So $\mu$ must be order preserving.

(3) $\mu$ is onto $X$. Suppose $y \in X$ and $\alpha \in I$. Because $\alpha$ is onto, there exists $x_{\alpha} \in X$ such that $\alpha\left(x_{\alpha}\right)=y$. Since $\Gamma \subseteq \mathscr{T}$, the elements of $\Gamma$ are ordered and, moreover, they are uncrossed. Thus, if $\alpha \succ^{\prime} \beta$, then since $\beta\left(x_{\beta}\right)=y=\alpha\left(x_{x}\right)>\beta\left(x_{x}\right)$ we see by the fact $\beta$ is order preserving, $x_{\beta} \succ x_{x}$. Since $\Gamma$ is bounded, let $\gamma$ be an upper bound, and so $\left\{x_{\alpha}: \alpha \in \Gamma\right\}$ is bounded from below by $x_{\eta}$. Let $x$ be the g.l.b. Since $x_{\alpha} \succsim x$, we see that $y=\alpha\left(x_{x}\right) \succsim \alpha(x)$. Thus, $y$ is an upper bound of $\{\alpha(x): \alpha \in \Gamma\}$, and so $y \succsim \mu(x)$. Suppose $y \succ \mu(x)$. By idempotence and monotonicity, the structure is intern and so $y \circ \mu(x) \succ \mu(x)$. By lower semicontinuity (Lemma 4.1 and the fact a closed intern structure is order dense), there exist $u, v \in X$ such that $u<y, v<\mu(x)$ and $u \circ v \succ \mu(x)$. So we may select $\alpha \in \Gamma$ such that $u \prec \alpha(x) \prec y$ and $v \prec \alpha(x) \prec \mu(x)$, whence

$$
\alpha(x)=\alpha(x) \circ \alpha(x) \succ u \circ v>\mu(x),
$$

which is a contradiction. So $\mu(x)=y$, proving $\mu$ is onto.

Thus, $\mu$ is an automorphism. We next show that it is in $\mathscr{T}$. Suppose for some $x \in X, \mu(x)=x$. If $\mu \neq i$, then for some $y \neq x, \mu(y) \neq y$. Suppose $\mu(y)>y$, then for some $\alpha \in \Gamma, \alpha(y)>y$. By the fact that $\mathscr{G}$ is uncrossed, $\alpha(x)>x$, and so $\mu(x) \succsim$ $\alpha(x) \succ x$, contrary to assumption. So $\mu(y) \prec y$. Since $\mathscr{X}$ is solvable, there exists $z \in X$ such that $x=y \circ z$ and $\mu(z) \lesssim z$. So, by the fact $\mu$ is an automorphism and using monotonicity,

$$
x=\mu(x)=\mu(y \circ z)=\mu(y) \circ \mu(z) \prec y \circ z=x,
$$

which is a contradiction. So $\mu \in \mathscr{T}$.

Suppose $\mu, \mu^{\prime}$ are, respectively, completions of $\Gamma, \Gamma^{\prime} \subseteq \mathscr{G}$. We show $\mu \mu^{\prime}$ is a completion. Observe,

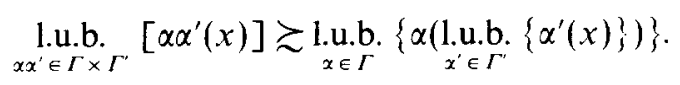

Suppose the inequality holds, then for some $\beta \in \Gamma$ and $\beta^{\prime} \in \Gamma^{\prime}$,

$$
\begin{aligned}
\beta \beta^{\prime}(x) & \succ \underset{\alpha \in \Gamma}{\text { l.u.b. }}\left(\alpha\left\{\underset{\alpha^{\prime} \in \Gamma^{\prime}}{\text { l.u.b. }}\left[\alpha^{\prime}(x)\right]\right\}\right) \\
& \succsim \text { l.u.b. }\left\{\alpha \beta^{\prime}(x)\right\} \\
& \gtrsim \beta \beta^{\prime}(x),
\end{aligned}
$$

which is a contradiction. Thus, $\mu \mu^{\prime}$ is the l.u.b. of $\Gamma \times \Gamma$, and so it is in $\mathscr{G}^{\prime}$. 
Next we show that if $\mu$ is the completion of a bounded subset $\Gamma$ of $\mathscr{G}$, then there is a bounded subset $\Gamma^{\prime}$ of $\mathscr{G}$ such that $\mu^{-1}$ is the completion of $\Gamma^{\prime}$. Let

$$
\Gamma^{\prime}=\left\{\beta: \beta \in \mathscr{G} \text { and, for all } \alpha \in \Gamma, \beta<\alpha^{-1}\right\} .
$$

Since $\Gamma$ has an upper bound $\gamma$, then $\gamma^{-1} \in \Gamma^{\prime}$. Moreover, $\Gamma^{\prime}$ is bounded by construction. Let $\mu^{\prime}$ be the completion of $\Gamma^{\prime}$. Suppose $\mu^{-1} \succ^{\prime} \mu^{\prime}$, then for each $\alpha \in \Gamma$, $\alpha^{-1} \succ^{\prime} \mu^{-1} \succ^{\prime} \mu^{\prime}$, and so $\mu^{-1} \in \Gamma^{\prime}$, which contradicts that $\mu^{\prime}$ is its l.u.b. Suppose $\mu^{-1} \prec^{\prime} \mu^{\prime}$, then $\mu \succ^{\prime} \mu^{\prime-1}$, and so there exists $\alpha \in \Gamma$ such that $\mu \succ^{\prime} \alpha \succ^{\prime} \mu^{\prime-1}$. Thus, $\mu^{\prime} \succ^{\prime} \alpha^{-1}$, contrary to choice.

So we have shown that the set of all completions forms a group lying between $\mathscr{G}$ and $\mathscr{T}$. Since $\mathscr{G}$ is a maximal group included in $\mathscr{T}$, it follows that $\left\langle\mathscr{G}, \succsim^{\prime}\right\rangle$ is Dedekind complete.

Combining Theorems 3.1 and 4.1, we see that a sufficient condition for homogeneity in the closed, idempotent, solvable, Dedekind complete concatenation structure is the existence of a maximal subgroup of $\mathscr{T}$ that is dense in $\mathscr{A}$. This fact is used in the proof of Theorem 4.3.

\subsection{Criteria for Homogeneity}

The first criterion for homogeneity is quite indirect. The second is more direct.

THEOREM 4.2. Suppose $\mathscr{X}$ is a concatenation structure that is closed, idempotent, solvable relative to some $x \in X$, Dedekind complete, and $N$-point unique for some integer $N$. Then $\mathscr{X}$ is homogeneous and solvable relative to each $x \in X$ iff all of the induced total concatenation structures (see Theorem 5.2 of Luce \& Narens, 1985) are isomorphic.

Proof. Suppose, first, that $\mathscr{X}$ is solvable relative to $x$ and is homogeneous, and let $y \in X$. By homogeneity there is an automorphism $\alpha$ such that $\alpha(x)=y$. For any $z \in X$, let $w$ solve $w \circ x=\alpha^{-1}(z)$, then

$$
z=\alpha \alpha^{-1}(z)=\alpha(w \circ x)=\alpha(w) \circ \alpha(x)=\alpha(w) \circ y,
$$

showing that $\alpha(w)$ is the solution relative to $y$. By Alper's (1984) theorem, the translations of $\mathscr{X}$ form a homogeneous subgroup. Thus, by Theorem 5.2 of Luce and Narens (1985) all of the induced total concatenation structures are isomorphic. The converse is immediate from the same theorem.

DefinItion 4.2. Suppose $\mathscr{X}$ is a closed concatenation structure. For $x, y \in X$ and $n$ an integer, define

$$
\theta(x, y, n)= \begin{cases}x \circ y & \text { if } \quad n=1 \\ \theta(x, y, n-1) \circ y & \text { if } \quad n>1\end{cases}
$$


THeOREM 4.3. Suppose $\mathscr{X}$ is a concatenation structure that is closed, idempotent, solvable, and Dedekind complete. Then $\mathscr{A}$ is homogeneous iff $\mathscr{T}$ is a group and there exists some $\tau \in \mathscr{T}, \tau \neq l$, such that for each integer $n, \theta(\tau, l, n), \theta\left(\tau^{-1}, l, n\right) \in \mathscr{T}$.

Proof. Necessity. By Theorems 2.1 and 5.1 of Luce and Narens (1985), $\mathscr{X}$ is isomorphic to a real representation and is 2-point unique, and by unboundedness (due to solvability) and Dedekind completeness of $\mathscr{X}$ the representation may be chosen to be onto $\mathrm{Re}^{+}$. By Alper's theorem, $\mathscr{T}$ is a group.

Since $\mathscr{X}$ is homogeneous, then Theorems 3.9, 3.12, and 3.13 of Luce and Narens (1985) show it has a unit representation $\mathscr{R}=\left\langle\mathrm{Re}^{+}, \geqslant, 0, f\right\rangle$. With no loss of generality, we assume $\mathscr{X}=\mathscr{R}$. Choose any $\tau \in \mathscr{T}$ with $\tau \succ l$; hence for some $c>1$, $\tau(x)=c x$. Let $\tau^{\prime}=\tau \circ I$, and observe that

$$
\tau^{\prime}(x)=\tau(x) \circ x=x f[\tau(x) / x]=x f(c x / x)=x f(c)=c^{\prime} x,
$$

and so $\tau^{\prime}$ is also a similarity (translation) with $c^{\prime}=f(c)>f(1)=1$. By induction, $\theta(\tau, l, n) \in \mathscr{T}$. The other case is similar.

Sufficiency. We establish this by first establishing two lemmas.

Lemma 4.2. Suppose $\mathscr{X}$ is a concatenation structure that is closed, idempotent, Dedekind complete, and upper (lower) semicontinuous. Then for each $x, y, z \in X$ with $x>z>y$, there exists an integer $n[m]$ such that $\theta(x, y, n) \prec z[\theta(y, x, m) \succ z]$.

Proof. Observe that $\{\theta(x, y, n): n$ an integer $\}$ is bounded from below by $y$ and so, by Dedekind completeness, there is a g.l.b. $w$. If $w=y$ we are done, so suppose $w \succ y$. Since $\mathscr{X}$ is intern, $w \succ w \circ y \succ y$. By upper semicontinuity, there exists $w^{\prime} \succ w$ such that $w \succ w^{\prime} \circ y$. By the choice of $w$, there exists an $n$ such that $\theta(x, y, n) \prec w^{\prime}$, in which case

$$
\theta(x, y, n+1)=\theta(x, y, n) \circ y \prec w^{\prime} \circ y \prec w,
$$

which is contrary to the choice of $w$. So $w=y$. The other case is similar.

COROLlary. Under the conditions of the theorem, the assertion of the lemma holds.

Proof. Lemmas 4.1 and 4.2.

LEMMA 4.3. Under the conditions of the Theorem, if for some $\tau \in \mathscr{T}, \tau \neq l$, and each integer $n, \theta(\tau, l, n), \theta\left(\tau^{-1}, l, n\right) \in \mathscr{T}$, then $\mathscr{T}$ is dense in $\mathscr{X}$.

Proof. Suppose $x>y$. If $x>\tau(y)>y$, we are done, so suppose $\tau(y) \gtrsim x>y$. By the Corollary to Lemma 4.2, there exists an $n$ such that $x>\theta[\tau(y), y, n]>y$, which yields half of $\mathscr{T}$ being dense in $\mathscr{X}$. The other half, which uses $\theta\left[\tau^{-1}(x), x, n\right]$, is similar.

Continuing the proof of sufficiency, since $\mathscr{T}$ is a group by Theorem 4.1, $\left\langle\mathscr{T}, \succsim^{\prime}\right\rangle$ is Dedekind complete and, by Lemma $4.2, \mathscr{T}$ is dense in $\mathscr{X}$. Thus, by Theorem 3.1, $\mathscr{A}$ is homogeneous. 
To use this result, one must first find a nontrivial translation $\tau$ of $\mathscr{X}$, and then verify, for each integer $n$, that $\theta(\tau, l, n)$ and $\theta\left(r^{-1}, l, n\right)$ are in $\mathscr{T}$. It is easy to see, using the unit representation, that if this is true for some $\tau$ of $\mathscr{T}$, then it is true for every $\tau$ in $\mathscr{T}$. So it does not matter which member of $\mathscr{T}$ one begins with. Note that property (ii) of the theorem is a countable set of conditions that closely resembles the property of each $n$-copy operator of a PCS being an automorphism (=translation). In fact, the PCS result could be stated as: $\imath \circ \imath \in \mathscr{T}=\mathscr{A}$ and, for each positive integer $n, \theta(l \circ l, l, n) \in \mathscr{T}$. The major difference in the two results is that in the idempotent case we do not have a specified automorphism with which to begin the induction.

The property of Theorem 4.3 is guaranteed when $\mathscr{X}$ is bisymmetric provided that $\tau \circ l$ is an onto map. This follows since $\tau \circ l$ clearly preserves $\succsim$ and it is easy to show that it preserves $\circ$,

$$
\begin{array}{rlrl}
(\tau \circ \imath)(x \circ y) & =\tau(x \circ y) \circ(x \circ y) & \\
& =[\tau(x) \circ \tau(y)][x \circ y) & & (\tau \in \mathscr{T}) \\
& =[\tau(x) \circ x] \circ[\tau(y) \circ y] & & \text { (bisymmetry) } \\
& =(\tau \circ \imath)(x) \circ(\tau \circ \imath)(y) . & &
\end{array}
$$

Thus, $\tau \circ \imath$ is an automorphism. In fact, it is a translation since were it not, then for some $x,(\tau \circ x)(x)=\tau(x) \circ x=x=x \circ x$, so by monotonicity, $\tau(x)=x$, contrary to choice.

The major improvement to be desired in Theorem 4.3 is to present explicitly one nontrivial translation or to prove that such an explicit formulation is impossible. The following argument suggests that a structural characterization of a single translation is not possible. As Narens (1981a) has discussed, it can be argued that any concept that can be defined in terms of the primitives of a structure must be invariant under transformations by the automorphisms of that structure. As he pointed out in part 5 of Theorem 3.3 of that paper, a necessary and sufficient condition for an automorphism to be invariant is that it commute with every automorphism. That obtains in the ratio scale case, but not in any others. So it seems doubtful if an explicit member of $\mathscr{T}$ can be described in general, although, of course, it may be easy to do so for particular structures.

\section{Existence of Dual Bilinear Representations}

\subsection{Background}

Pfanzagl (1959(a), 1959(b)) established the existence of wcighted avcrage representations for idempotent, bisymmetric concatenation structures $\mathscr{X}=$ $\langle X, \succsim, \circ\rangle$. Specifically, he proved the existence of a mapping $\varphi$ from $X$ into the real numbers, Re, and a constant $c, 0<c<1$, such that $\varphi$ is order preserving and

$$
\varphi(x \circ y)=c \varphi(x)+(1-c) \varphi(y) .
$$


This is an interval or $(2,2)$ scale type. A derivation of the result, which depends upon additive conjoint measurement, can be found in Section 6.9 of Krantz et al. (1971).

The interest in such structures stems from the existence of empirical averaging operations. Perhaps the one best known in the social sciences is subjective expected utility theory, where o represents the mixing of two gambles by some chance event, and so $c$ can be thought of as a weight (possibly a probability) associated with the event.

Luce and Narens $(1983,1985)$ have pointed out that this representation is a spccial case of a more general interval scale representation, namely that there exist two constants $c$ and $d$, both in $(0,1)$, such that

$$
\varphi(x \circ y)= \begin{cases}c \varphi(x)+(1-c) \varphi(y) & \text { if } \quad x \succ y \\ \varphi(x) & \text { if } \quad x=y \\ d \varphi(x)+(1-d) \varphi(y) & \text { if } \quad x<y\end{cases}
$$

They have referred to this as the dual bilinear representation. Note that it too is invariant under any affine transformation and so is of scale type $(2,2)$. Moreover, as they show, it is the most general representation of a concatenation structure of that type onto the real numbers. Also, Luce and Narens (1985) develop from it a generalization of subjective expected utility which is not inconsistent with some of the empirical phenomena that have been found to be. inconsistent with the classical theory. Basically, the underlying qualitative theory invokes only a very limited form of rationality; once rationality is extended to more complex gambles, the classical theory results.

A natural question to raise is: under what qualitative conditions does the representation of Eq. (2) exist, i.e., what is the generalization of Pfanzagl's representation by Eq. (1). An answer is provided. It may not be regarded as fully satisfactory because it is formulated in terms of defined operations. In principle, the axioms may be translated back into the primitives, but to do so results in a rather messy system that would be difficult to understand. The two defined operations are the qualitative analogues of the two operations obtained from Eq. (2) by assuming the constant $c$ works throughout, as in Eq. (1), and by assuming the constant $d$ works throughout. So the one operation, denoted $*$, coincides with $\circ$ for $x \succsim y$, and extends it throughout the domain, and the other, denoted $*^{\prime}$, coincides with $\circ$ for $x \lesssim y$, and extends that part throughout the domain. As we shall see, these defined operations are not as objectionable as they might be since they do not rest on entities that are particularly difficult to find.

\subsection{Formulation of the Result}

The basic observation that we use is that the equation

$$
(u \circ x) \circ(v \circ y)=(u \circ v) \circ w
$$


is independent of $u$ and $v$ in a bisymmetric structure since by bisymmetry and monotonicity,

$$
w=x \circ y .
$$

In a dual bisymmetric structure, the same independence follows so long as inequalities are maintained to keep everything on one side of the bilinear structure. For example, suppose $u>x, v>y, u \circ v>v \circ y, u>v$, and $u \circ v>w$, then by Eq. (2), we see

$$
\begin{aligned}
& c[c \varphi(u)+(1-c) \varphi(x)]+(1-c)[c \varphi(v)+(1-c) \varphi(y)] \\
& \quad=c[c \varphi(u)+(1-c) \varphi(v)]+(1-c)[c \varphi(x)+(1-c) \varphi(y)] \\
& \quad=c[c \varphi(u)+(1-c) \varphi(v)]+(1-c) \varphi(w) .
\end{aligned}
$$

Thus, by canceling the $u$ and $v$ term, we have

$$
\varphi(w)=c \varphi(x)+(1-c) \varphi(y) .
$$

Of course, this is nothing new when $x \succ y$, but when $x<y$ it suggests a way to extend the operation $\circ$ from $x>y$ to $x \prec y$ so that bisymmetry holds for the new operation.

To do so, we must first establish that a suitable $w$ always exists and then impose a condition corresponding to its invariance under the choice of $u$ 's and $v$ 's satisfying the necessary inequalities.

Lemma 5.1. Suppose $X$ is a concatenation structure that is idempotent and solvable. For each $x, y \in X$,

(i) if $x \prec y$, then there exist $u, v, w \in X$ such that

$$
u \succsim x, \quad v \gtrsim y, \quad u \circ x \succsim v \circ y, \quad u \circ v \succsim w,
$$

and

$$
(u \circ x) \circ(v \circ y)=(u \circ v) \circ w
$$

(ii) if $x>y$, then there exist $p, q, z \in X$ such that

$$
p \lesssim x, \quad q \lesssim y, \quad p \circ x \lesssim q \circ y, \quad p \circ q \lesssim z,
$$

and

$$
(p \circ x) \circ(q \circ y)=(p \circ q) \circ z .
$$

Proof. Since the two proofs are similar, only (i) is presented. By the nontrivialness of the structure and solvability, select $u$ and $v$ so that $u \gtrsim x, v \succsim y$, and $u \circ x \succsim v \circ y$. Define $w$ as the solution to Eq. (4). It is now sufficient to show the last 
inequality of Eq. (3). Suppose, on the contrary, $u \circ v<w$, then using the idempotency and monotonicity of $\circ$,

$$
u \circ v=(u \circ v) \circ(u \circ v) \succsim(u \circ x) \circ(v \circ y)=(u \circ v) \circ w \succ(u \circ v) \circ(u \circ v)=u \circ v,
$$

a contradiction.

COROLlary. If Eq. (3) holds, then $u \succsim v$.

Proof. Since $y \succ x$ and $u \circ x \succsim v \circ y$, monotonicity yields $u \circ x \succsim v \circ x$, and so $u \succsim v$.

Thus, we may define $*$ and $*^{\prime}$ that partially coincide with $\circ$ as follows: for each $x$, $y \in X$,

$$
\begin{aligned}
& x * y= \begin{cases}x \circ y & \text { if } \quad x>y, \\
x & \text { if } \quad x=y, \\
w & \text { if } x<y, \text { where } w \text { is the element of Lemma 5.1, }\end{cases} \\
& x * y=\left\{\begin{array}{lll}
z & \text { if } x>y, \text { where } z \text { is the element of Lemma 5.1. } \\
x & \text { if } x=y, \\
x \circ y & \text { if } x<y .
\end{array}\right.
\end{aligned}
$$

In order that $*$ and $*^{\prime}$ be well-defined operations, it is essential that Eq. (4) (Eq. (6)) not depend on the choice of $u$ and $v$ ( $p$ and $q$ ) so long as Eq. (3) (Eq. (5)) is satisfied. This and the needed interness of these operations is captured in the next definition.

Definition 5.1. Suppose $\mathscr{X}$ is a concatenation structure. Then is said to be reflectable iff for each $x, y, w, z \in X$,

(i) if $x<y$ and Eqs. (3) and (4) hold for some $u, v \in X$, then Eq. (4) holds for all $u, v, w$ satisfying Eq. (3), and $x<w<y$;

(ii) if $x \succ y$ and Eqs. (5) and (6) hold for some $p, q \subset X$, then Eq. (6) holds for all $p, q, z$ satisfying Eq. (5), and $x>z>y$.

LEMMA 5.2. Suppose $\mathscr{X}$ is a concatenation structure that is idempotent, solvable, and reflectable. Then $*$ and $*^{\prime}$ defined by Eqs. 7 and 8 are well-defined operations that are idempotent, intern, monotonic, and solvable.

Proof. As the two cases are symmetric, it suffices to prove the result for *. By reflectableness, $*$ is a well-defined operation that is intern, and by construction it is idempotent.

Next we show that $*$ is monotonic. 
(i) If $x, y \succsim z$, then $x * z=x \circ z$ and $y * z=y \circ z$, and the monotonicity of * follows from that of $\circ$.

(ii) If $x \succ z \succ y$, then from the fact that $*$ is intern $x * z=x \circ z \succ z \succ y * z$. The case $x \prec z \prec y$ is similar.

(iii) If $z \lesssim x, y$, then select $v \in X$ such that $v \succ z$, and let $u, u^{\prime} \in X$ solve $u \circ x=$ $v \circ z=u^{\prime} \circ y$. Since $z \lesssim x, y$, it follows by monotonicity of $\circ$ that $u, u^{\prime} \prec z \lesssim x, y$, and by interness of $*, u \circ v<x * z$ and $u^{\prime} \circ v<y * z$. Thus, by reflectability,

$$
(u \circ x) \circ(v \circ z)=(u \circ v) \circ(x * z) \quad \text { and } \quad\left(u^{\prime} \circ y\right) \circ(v \circ z)=\left(u^{\prime} \circ v\right) \circ(y * z) \text {. }
$$

Since $u \circ x=u^{\prime} \circ y$, monotonicity yields

$$
(u \circ v) \circ(x * z)=\left(u^{\prime} \circ v\right) \circ(y * z) .
$$

Using the monotonicity of $\circ$ and this relation, we see

$$
\begin{array}{ll}
x \succsim y \quad \text { iff } \quad u \lesssim u^{\prime} \quad\left(\text { since } u \circ x=u^{\prime} \circ y\right) \\
& \text { iff } \quad u \circ v \precsim u^{\prime} \circ v \\
& \text { iff } \quad x * z \succsim y * z .
\end{array}
$$

The other side is similar.

Finally, we show that $*$ is solvable. Suppose $x$ and $z$ are given, and we search for $y$ such that $z=x * y$. (The existence of the solution $y$ to $z=y * x$ is shown similarly.) If $x \succsim z$, then let $y$ solve $z=x \circ y$. Since by monotonicity, $x \succsim y$, we see $x \circ y=x * y$. So, suppose $x \prec z$. Select $u$ to be any element for which $u \succ x$ and $u \circ x>z$. Select $p$ so that $u \circ x>p>z$. Let $w$ solve $(u \circ x) \circ p=w \circ z, v$ solve $u \circ v=w$, and $y$ solve $v \circ y=p$. Observe, by monotonicity and transitivity

$$
(u \circ x) \cup(v \circ y)-(u \circ x) \circ p=w \circ z=(u \circ v) \circ z,
$$

and so by reflectability, $z=x * y$ provided the requisite inequalities hold. We have $u \succ x$ and $u \circ v \succ p=v \circ y$ by choice. Since $w \circ z=(u \circ x) \circ p \succ z \circ z=z$, we see $u \circ v=$ $w \succ z$. Finally, we show $v \succsim y$. From $v \circ y=p, v \succsim y$ iff $v \succsim p$. Suppose $v \prec p$, then

$$
w=u \circ v<u \circ p<(u \circ x) \circ p=w \circ z,
$$

whence $w \prec z$, contrary to what was shown above.

An operation is said to be right autodistributive iff for all $x, y, z \in X$,

$$
(x \circ y) \circ z=(x \circ z) \circ(y \circ z) .
$$

Two operations $*$ and $*^{\prime}$ on $X$ are said to satisfy generalized bisymmetry iff for all $u, v, x, y \in X$,

$$
(u * v) *^{\prime}(x * y)=\left(u *^{\prime} x\right) *\left(v *^{\prime} y\right)
$$


THEOREM 5.1. Suppose $\mathscr{X}$ is a (totally ordered) concatenation structure that is idempotent, solvable, and Dedekind complete. Then $\mathscr{X}$ has a dual bilinear representation (Eq. (2)).iff $\mathscr{X}$ is reflectable (Definition 5.1), * and *' defined by Eqs. (7) and (8) are each right autodistributive (Eq. (9)), and they satisfy generalized bisymmetry (Eq. (10)).

The rest of the paper is devoted to the proof, one part of which is a result about functional equations, to which we turn first.

\subsection{Proof of a Lemma about Functional Equations}

LEMmA 5.3. Suppose that $F$ and $G$ are functions from $\operatorname{Re} \times \operatorname{Re}$ onto $\operatorname{Re}$ satisfying the following properties: for some strictly increasing function $g$ from $\operatorname{Re}$ onto $\operatorname{Re}$ with $g(0)=0$ and $g(1)=1$ and constants $c, d \in(0,1)$, and for all $u, v, x, y \in \operatorname{Re}$,

$$
\begin{aligned}
F(x, y) & =c x+(1-c) y, \\
G(x, y) & =g^{-1}[d g(x)+(1-d) g(y)], \\
F[G(u, v), G(x, y)] & =G[F(u, x), F(v, y)],
\end{aligned}
$$

then $g$ is the identity.

Proof. According to Aczél (1966, p. 317), the general solution to Eq. (13) is of the form

$$
\begin{aligned}
& F(x, y)=k^{-1}[A h(x)+B h(y)+C], \\
& G(x, y)=m[A k(x)+B k(y)+C],
\end{aligned}
$$

where $h, k$, and $m$ are strictly increasing functions. Since by Eqs. (11) and (12), $F(x, x)=x=G(x, x)$, it follows from Eqs. (14) and (15) that

$$
\begin{aligned}
k(x) & =(A+B) h(x)+C, \\
m^{-1}(x) & =(A+B) k(x)+C .
\end{aligned}
$$

From Eqs. (11), (14), and (16),

$$
\begin{aligned}
A h(x)+B h(y)+C & =k F(x, y) \\
& =k[c x+(1-c) y] \\
& =(A+B) h[c x+(1-c) y]+C .
\end{aligned}
$$

By Aczél (1966, p. 67), Eq. (18) implies that $h$ is linear, and by Eqs. (16) and (17), so are $k$ and $m$. From this and Eqs. (12) and (15),

$$
\begin{aligned}
c g(x)+(1-c) g(y) & =g[G(x, y)] \\
& =g\{m[A k(x)+B k(y)+C]\},
\end{aligned}
$$

from which it follows that $g$ is linear. Since $g(0)=0$ and $g(1)=1$, it follows that $g$ is the identity. 


\subsection{Proof of Theorem 5.1}

By Theorems 2.1 and 5.1 of Luce and Narens (1985), $X$ has a real representation which, because $\mathscr{X}$ is unbounded, is onto Re. So, with no loss of generality, we may suppose that $\circ, *$ and $*^{\prime}$ are all real operations. First, suppose $\circ$ has a dual bilinear form (see Eq. (2)). Let - be the operation defined by: for all $x, y \in X, x \backsim y=$ $c x+(1-c) y$. We show that $*=\mathbf{m}$. If $x \geqslant y$, they agree by definition. For $x<y$, select $u, v$ in $X$ so that $u>x, v>y$, and $u \circ x>v \circ y$, and so

$$
(u \circ x) \circ(v \circ y)=(u \circ v) \circ(x * y) .
$$

By the dual bilinear form of $\circ$ and keeping the inequalities in mind, we have

$$
\begin{gathered}
{[c u+(1-c) x] c+[c v+(1-c) y](1-c)} \\
=[c u+(1-c) v] c+(x * y)(1-c) .
\end{gathered}
$$

Solving,

$$
x * y=c x+(1-c) y=x \boldsymbol{\bullet} .
$$

In like manner,

$$
x \boldsymbol{\bullet}^{\prime} y=d x+(1-d) y=x *^{\prime} y .
$$

It is trivial to verify that $\boldsymbol{\omega}$ and $\boldsymbol{\boldsymbol { a }}^{\prime}$, and hence $*$ and $*^{\prime}$, are bisymmetric and satisfy generalized bisymmetry. Right autodistributivity is a special case of bisymmetry.

Conversely, by Theorem 6.4 of Luce and Narens (1985), under these conditions the right autodistributivity of $*$ and $*^{\prime}$ imply that they are bisymmetric. So there is no loss of generality in assuming $*$ has the representation

$$
x * y=c x+(1-c) y .
$$

Since $*^{\prime}$ also has a bisymmetric representation, there is a strictly increasing function $g$ such that

$$
x *^{\prime} y=g^{-1}[d g(x)+(1-c) g(y)],
$$

and we may select $g(0)=0$ and $g(1)=1$. If we set $F(x, y)=x * y$ and $G(x, y)=$ $x *^{\prime} y$ and note that the dual bisymmetry of $*$ and $*^{\prime}$ is equivalent to Eq. (13) of Lemma 5.3 and Eqs. (19) and (20) are equivalent to Eqs. (11) and (12), we conclude that $g$ is the identity. This yields the dual bisymmetric representation because of the relation between $\circ$ and $*^{\prime}$.

\section{ConCluding Remarks}

The following problems need to be solved. First, provide examples to show that the hypotheses are really needed for the theorems. Second, characterize the trans- 
formation groups of automorphisms for general non-homogeneous, but finitely unique structures. This may only be interesting when some structure is imposed on the automorphism group. Third, try to show directly under the assumptions of Theorem 3.3, that the dilations are Archimedean and so, by Theorem 2.6, members of the commutator subgroup are translations. Fourth, formulate, or prove impossible, an explicit definition of a maximal subgroup of translations of an idempotent structure in terms of the primitives. And fifth, formulate representation theorems, analogous to those for homogeneous PCS and the $(2,2)$ case, for the idempotent $(1,1)$ and $(1,2)$ cases.

\section{REFERENCES}

ACzÉL, J. (1966). Lectures on functional equations and their applications. New York: Academic Press.

ALPER, T. M. (1984). Groups of homeomorphisms of the real line. B.S. Thesis, Cambridge, MA: Harvard University.

AlPER, T. M. (1985). A note on real measurement structure of scale type $(m, m+1)$. Journal of Mathematical Psychology, 29, 73-81.

Burkenhout, F., \& Hubaut, X. (1966). Groupes affins et projectifs. Bulletin de l'Académie Royale de Belgique, Classe des Sciences, 52, 368-381.

COHEN, M. \& Narens, L. (1979). Fundamental unit structures: A theory of ratio scalability. Journal of Mathematical Psychology, 20, 193-232.

Fuchs, L. (1963). Partially ordered algebraic systems. Reading, MA: Addison-Wesley.

Krantz, D. H., Luce, R. D., Suppes, P., \& TVersky, A. (1971). Foundations of measurement (Vol. 1). New York: Academic Press.

Levine, M. V. (1970). Transformations that render curves parallel. Journal of Mathematical Psychology, $7,410-443$.

LEviNE, M. V. (1972). Transforming curves into curves with the same shape. Journal of Mathematical Psychology, 9 1-16.

LuCE, R. D. \& NARENS, L. (1983). Symmetry, scale types, and generalizations of classical physical measurement. Journal of Mathematical Psychology, 27, 44-85.

LuCE, R. D. \& NARENS, L. (1985). Classification of concatenation measurement structures according to scale type. Journal of Mathematical Psychology, 29 1-72.

NARENS, L. (1981(a)). A general theory of ratio scalability with remarks about the measurementtheoretic concept of meaningfulness. Theory and Decision, 13, 1-70.

NARENS, L. (1981(b)). On the scales of measurement. Journal of Mathematical Psychology, 24, 247-275.

NARENs, L. \& LuCE, R. D. (1976). The algebra of measurement. Jounal of Pure and Applied Algebra, 8, 197-233.

Pfanzagl, J. (1959(a)). Die axiomatischen Grundlagen einer allgemeinen Theorie des Messens. Schrift. Stat. Inst. Univ. Wien, Neue Folge, Nr. 1. Wurzburg: Physica-Verlag.

Pfanzagl, J. (1959(b)). A general theory of measurement-Applications to utility. Naval Research Logistics Quarterly, 6, 283-294.

TIrs, J. (1952(a)). Généralisations des groupes projectifs basées sur leurs propriétés de transitivité. Mémoires de l'Académie Royale de Belgique, Classe des Sciences, Tome XXVII, Fascicule 2.

TrTs, J. (1952(b)). Sur les groupes doublement transitifs continus. Commentarii Mathematicia Helveticic, 26, 203-224.

ReCeived: August 5, 1985 